\title{
Fog Computing-based Traffic Management Support forIntelligent Transportation Systems
}

\section{Celso A. R. L. Brennand ${ }^{13}$, Daniel Ludovico Guidoni ${ }^{2}$ (Co-advisor), Leandro Aparecido Villas ${ }^{1}$ (Advisor)}

\author{
${ }^{1}$ Institute of Computing, University of Campinas, Brazil \\ ${ }^{2}$ Department of Computer Science, Federal University of São João del-Rei, Brazil \\ ${ }^{3}$ UAST, Federal Rural University of Pernambuco, Brazil \\ celso.brennand@ufrpe.br, guidoni@ufsj.edu.br, leandrodic.unicamp.br
}

\begin{abstract}
Traffic in large urban centers contributes to problems ranging from decreasing the population's quality of life and security to increasing financial costs for people, cities, and companies. Considering the advance of communication, processing, and sensing technologies, Intelligent Transport Systems (ITS) have emerged as an alternative to mitigate these problems. The interoperability of ITS with new technologies, such as vehicular networks (VANETs) and Fog computing, make them more promising and effective. VANETs ensure that vehicles have the computing power and wireless communication capabilities providing a new range of security and entertainment services for drivers and passengers can be developed. However, these types of services, especially traffic management, demand a continuous analysis of vehicle flow conditions on roads. Thereby, a huge network and processing resources are required making the development of ITS solutions more complex and difficult to scale. Fog computing is a decentralized computing infrastructure in which data, processing, storage, and applications are distributed at the network edge, thereby increasing the system's scalability. In the literature, traffic management systems do not adequately address the scalability problem, resulting in load balancing and response time problems. This doctoral thesis proposes a traffic management system based on the Fog computing paradigm to detect, classify, and control traffic congestion. The proposed system presents a distributed and scalable framework that reduces the aforementioned problems in relation to state of the art. Therefore, using Fog computing's distributed nature, the solution implements a probabilistic routing algorithm that balances traffic and avoids the problem of congestion displacement to other regions. Using the characteristics of Fog computing, a distributed methodology was developed based on regions that collect data and classify the roads concerning the traffic conditions shared by the vehicles. Finally, a set of communication algorithms/protocols was developed which, compared with other literature solutions, reduces packet loss and the number of messages transmitted. The proposed service was compared extensively with other solutions in the literature regarding traffic metrics, where the proposed system was able to reduce downtime by up to $70 \%$ and up to $49 \%$ of the planning time index. Considering communication metrics, the proposed service can reduce packet collision by up to $12 \%$ reaching $98 \%$ coverage of the scenario.
\end{abstract}




\section{Introduction}

The unplanned development of urban centers is often associated with severe socioeconomic problems. Such uncontrolled urban growth typically causes significant stress on city structures due to the unexpected demand of various resources and services. One of the most affected sectors is urban transport systems, in which inefficiencies may lead to many negative consequences. Among them is the increase in greenhouse gas emissions and many hours stuck in traffic congestions, thus resulting in health issues and monetary losses. For instance, the congestion costs in the United States, the United Kingdom, and Germany were almost \$461 billion in 2017 [Cookson and Pishue 2017]. The INRIX 2019 Global Traffic Scorecard [Cookson and Pishue] shows that drivers from the city of Rio de Janeiro/Brazil spend an average of 190 hours per year on traffic congestion, and their average speed is $17 \mathrm{kph}$ (where 50-60kph is desire), occupying the 2 nd place in the ranking of the most congested cities in the world. This problem is generally generated by the increase in the number of vehicles and lack of public transportation investment to support this increase. This urban growth typically causes significant stress on city structures due to the increased demand for various resources and services, besides serious socioeconomic problems.

However, it is not always possible to improve the road network due to various factors such as space constraints to build new roads, environmental issues and local city policies. One approach to alleviate these problems is the development of an Intelligent Transport System (ITS). An ITS uses communication, processing and sensing technologies to improve urban traffic and, consequently the flow of vehicles in urban roads. Moreover, an ITS does not only aim to provide traffic management services (for instance, to prevent traffic jam) but also security management services and infotainment applications to drivers, passengers and pedestrians [Brennand et al. 2015, da Cunha et al. 2016, Villas et al. 2014]. The basis for an efficient ITS is the collaborative approach where each element of the system such as vehicles, sensors and mobile devices contributes by providing important information to the system [Papadimitratos et al. 2009]. In ITS, vehicles are equipped with sensors (e.g., GPS and Galileo), processors and wireless communication modules. Thus, vehicles can communicate with other vehicles through vehicle-to-vehicle (V2V) communication and with the network infrastructure (e.g., RSU-Road Side Unit) through vehicle-to-infrastructure (V2I) communication. Some ITS applications are road hazard warnings where the driver is warned of possible path problems, such as ice formation on roads, accident warnings, and route suggestion services.

ITS services and applications have intrinsic characteristics regarding the way they process, store and disseminate a vast amount of data generated in ITS [Rizzo et al. 2016, El-Sayed and Chaqfeh 2019]. These characteristics imply in some issues for ITS service requirements such as mobility, frequent network disconnections, networking latency, end-to-end response time and spatial context awareness. Thus, designing ITS services that have a required quality of service (QoS) is a challenging [Ai et al. 2018, El-Sayed and Chaqfeh 2019]. A traditional centralized architecture is not able to provide these requirements mainly due to the exponentially increasing number of vehicles and new services with very strict constraints of computational and network resources such as route suggestions and accident warning services. Thus, applying the Fog computing paradigm in ITS can solve its challenges. Fog computing is a decentralized computing paradigm where computing resources (processing/network/storage) are at the edge of the network. 
Thus, resources are located more logically, efficiently, and close to users/devices.

The main benefits to designing an ITS with Fog paradigm are [Bonomi et al. 2012]:

- Low latency-some ITS data have strict time constraints, such as data for re-route systems;

- Predominant wireless access-modern ITS systems heavily rely on wireless communications;

- Wide geographical distribution-ITS has geographically spread sensors. However, the scope of the data gathered is restricted to the location of the sensors that generated such data;

- Real-time interaction-re-routing systems have real-time requirements;

- Mobility-an ITS is used to optimize the mobility of vehicles in the city. However, the ITS may also leverage mobility to perform data delivery activities to various stakeholders;

- Scalability-an ITS needs to be scalable due to the high number of vehicles and sensors;

- and Extensibility-if the city grows, the ITS infrastructure also needs to grow to support the expanded region.

These characteristics enable Fog Computing to offer an ideal platform for a highly dynamic and heterogeneous ITS environment [El-Sayed and Chaqfeh 2019].

These characteristics enable Fog Computing to offer an ideal platform for a highly dynamic and heterogeneous ITS environment [El-Sayed and Chaqfeh 2019]. Generally, route suggestion services rely on data from specific regions, such as traffic conditions, which may be irrelevant to other regions of a city [Brennand et al. 2019]. In this scenario, this service may exchange a large amount of data from heterogeneous data sources [Rizzo et al. 2016, Wang et al. 2018] to monitor traffic conditions in a particular region. Moreover, the data may have real-time constraints and it can be disseminated using different communication technologies [Brennand et al. 2016] and considering the dynamic topology, frequent network disconnections and cooperative communication [Al-Sultan et al. 2014]. It is worth noticing that sending data to a single central entity (e.g., Cloud) is a waste of system resources, such as the network bandwidth. Moreover, data transmissions are more vulnerable to specific problems, such as delays, data loss, scalability, and communication disruption. Hence, route suggestion services in ITS's are not well suited to centralized architectures such as Cloud computing [Brennand et al. 2016, Ling et al. 2018, Wang et al. 2018, Kuang et al. 2019].

In this scenario, a route management service that takes advantage of the features of the Fog computing paradigm is extremely desirable in ITS. This happens because the Fog computing paradigm moves its resources (storage and processing) to the edge of the network, thus bringing the available resources as close as possible to end-users without the assistance of the Internet core [Bonomi et al. 2012]. The Fog computing paradigm is based on entities called Cloudlets which have processing and communication capabilities (e.g., micro-data centers) and are geographically distributed to be closer to the access networks [Satyanarayanan et al. 2009]. Since Cloudlet resources are closer to the end devices, they allow a faster response time and a local service decision. Thus, the Fog paradigm provides geo-computation and faster and less costly communication 
when compared to a Cloud. Although the Fog paradigm has lower computing capacity when compared with the Cloud, it can use Cloud data centers whenever necessary. Another limitation is that knowledge is collected and belongs to Cloudlet's region. Thus, a route management service, for example, need to share information between Cloudlet's to provide a reliable service.

This approach forms a multi-tier architecture (see Figure 1), which is hierarchically organized with varying types of capabilities and end-user proximity. Cloud computing, represented in Figure 1 Tier A, has a more powerful resource. However, the longer distance to retrieve data and the presence users beyond congested connections due to the use of the Internet often limit the real-time services and increase the network cost, especially considering a high dynamic vehicular network topology. Fog paradigm and Cloudlet environment are shown in Figure 1 Tier $\mathbf{B}$, where the resources are closer to the end-devices permitting a faster response time and a local service decision. Finally, user and sensor devices (e.g., vehicles, road sensors) are represented in Figure 1 Tier $\mathbf{C}$.

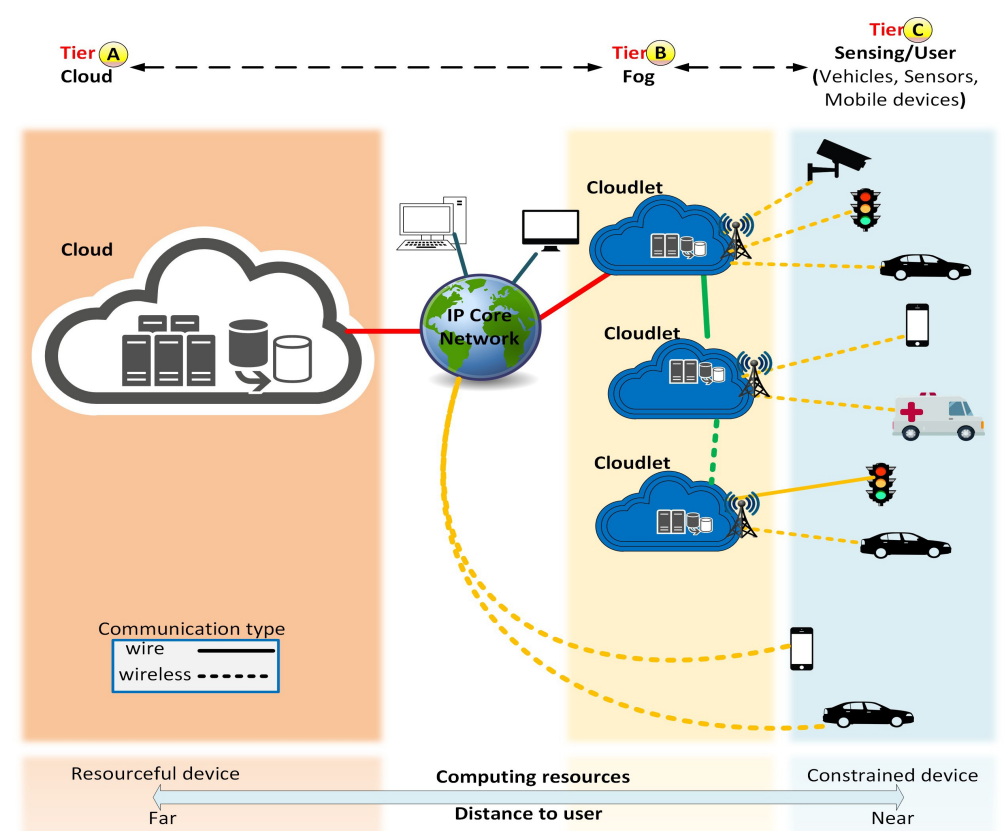

Figure 1. Cloud and Fog representation.

In the literature, several studies address the problem of route management in urban centers [Gomides et al. 2019, Jeong et al. 2016, de Souza et al. 2016]. Jeong et al. [Jeong et al. 2016] proposed a centralized solution for traffic optimization where road data is collected and sent to a central server that will classify the traffic and perform routing calculation, thus sending a new route to vehicles. However, as the processing is centralized and the communication uses the Internet, the network latency and system scalability are a problem. Gomides et al., [Gomides et al. 2019] present a fully distributed vehicle solution in which traffic motoring, classification, and route computation are made by vehicles on the system. However, VANET characteristics, such as data dissemination, hamper theses solutions acquiring full knowledge of the map containing the traffic information and characteristics of the environment (e.g., roads, maximum speed). Sousa [de Souza et al. 2016] present infrastructured distributed solutions that use RSUs 
to classify the traffic and compute the route. However, RSUs do not have full knowledge of the map because the RSU does not have communication between them.

In most of them, this kind of service uses an architecture for carrying out the monitoring and traffic control that rely on information about the vehicles, as well as the characteristics of the routes. However, these architectures also have to exchange, process and store a considerable amount of data generated by the devices that are embedded in vehicles and that are used for monitoring city traffic. Thus, problems related to processing (e.g., load balance, response time) and data transmissions (e.g., delays, data loss and communication disruption) become a concern. Besides, in route suggestion services, the response time to perform the decision-making process must be within an acceptable time frame so that the information is still useful in order for the vehicle's driver to carry out the necessary route changes.

Given the aforementioned limitations, the thesis proposes a framework solution named FOXS - Fast Offset Xpath Service. FOXS is based on the Fog computing paradigm for Intelligent Transport System services to detect, classify, and control traffic congestion. FOXS framework uses Cloudlets to monitor traffic conditions and to calculate the vehicle route. Thus, it enables computational power to reside closer to where it is most required, thus dividing the system load and increasing the overall scalability of the system and holding the capability to collect, process and store large volumes of data. To do this, FOXS framework uses the network infrastructure Road side Units (RSUs) as a Cloudlet entity, which is deployed in the city to manage the traffic of vehicles. For its operation, a mechanism that gathers all necessary data from vehicles and road sensors was developed. This mechanism optimizes the delivery rate and reduces the number of messages in the system. Also was developed a VANET multihop protocol based on routing service characteristics and requirements. Therefore, with the data collected by the corresponding Fog entities, the level of congestion of the roads is estimated. Finally, according to the conditions of the roads, the corresponding Fog entities calculate a new route as a suggestion.

The Flowchart 2 describes the basic behavior of FOXS. The stage Data Gathering is responsible for sensing the environment and sending the gathered data to the next stage, Data Processing. The data is collected by sensors in the vehicles (e.g., GPS, odometer, speed) and by infrastructured sensors (e.g., the density of vehicles in the region). This data is sent to the nearest RSU. In the Data Processing stage, the gathered data are processed and stored per each Cloudlet to classify the vehicle traffic congestion levels. As FOXS requirement, the processed data is shared with the nearby Cloudlets to generate sufficient traffic knowledge for Cloudlets. In the Service Delivery stage, each Cloudlet computes suggested routes to vehicles in its region when the service intervention is necessary (e.g., a vehicle travels to a congested region or to avoid the formation of a traffic jam). All communication between the vehicle and the RSU can occur through VANET directly or by multiple hops using the developed VANET protocol.

\section{Objectives and Contributions}

The main contribution of this thesis is a new framework to traffic management service based on the Fog computing paradigm. The aims are to:

- Design a set of algorithms and mechanisms to increase the packet delivery rate in the VANET environment and the RSU distribution algorithm; 


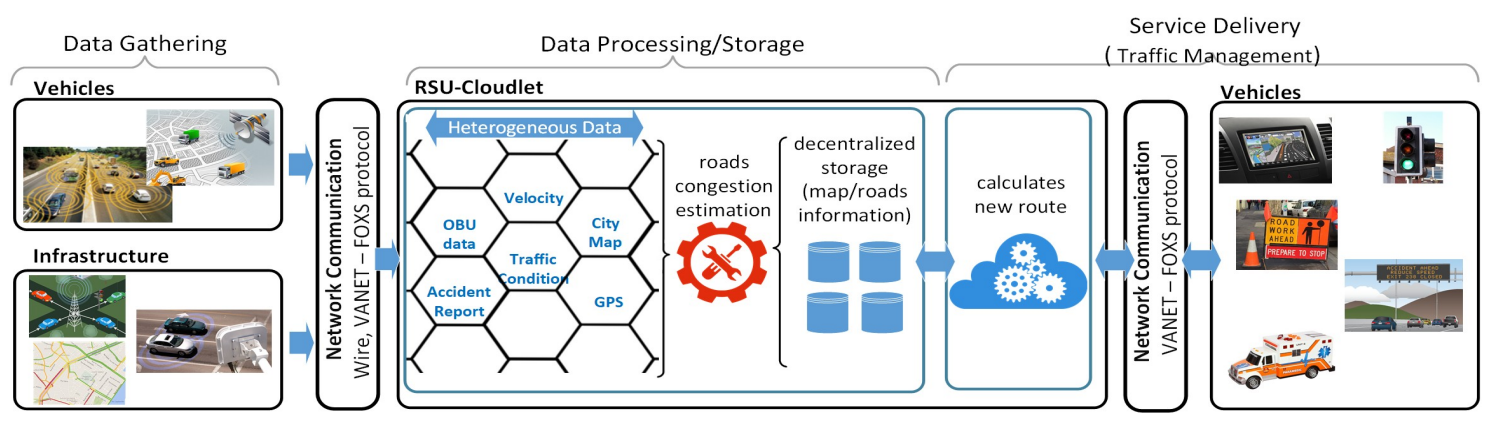

Figure 2. FOXS' flowchart.

- An algorithm/methodology for traffic classification;

- An algorithm to compute and choose better routes for vehicles;

- An ITS framework based on Fog computing to provide route traffic management services;

- A distributed protocol for multihop communication in VANET;

- Evaluate the proposed approach against state-of-art route traffic management services solutions.

The student has been published/submitted a number of papers. Among the publications, an award for the best paper was received.

\section{Best paper awards - In 2016 IEEE Symposium on Computers and Communication}

The first article related to the thesis was published in 2015 IEEE Symposium on Computers and Communication (ISCC '15) [Brennand et al. 2015]. This paper presents the probabilistic algorithm for suggesting routes for vehicles. The algorithm, probabilistically, suggests alternative routes to vehicles in a balanced way, avoiding the creation of new congestion. The service was evaluated in a simulated manner with different conditions and parameters to understand the behavior of the solution. The proposed solution reduced the average travel time by approximately $23 \%$ and the $\mathrm{CO} 2$ emission by $10 \%$.

Although the solution presented reduces congestion, problems inherent to scalability, low latency, and network conditions are not addressed. Thus, article Fox: $\boldsymbol{A}$ traffic management system of computer-based vehicles fog (IEEE-ISCC16-Best paper awards) [Brennand et al. 2016] proposes using the Fog computing paradigm to address these problems in the route suggestion service proposed. To use this paradigm, the vehicle routing algorithm was redesigned, thus allowing the sharing of information on road conditions between all Cloudlets. Thus, each Cloudlet can execute the algorithm independently and using only information necessary for vehicle routing in its region. In this article, simulation results show that the travel time reduced $32 \%$ and the stop time by $59 \%$.

The article published in 2017 IEEE Symposium on Computers and Communications (ISCC)[Brennand et al. 2017], in addition to improving the routing service, presents an assessment of the communication network and its impact on the proposed service. For network results, the packet collisions were reduced by up to $8 \%$.

The work published in International Journal of Distributed Sensor Networks[De Souza et al. 2017] presents a traffic management systems classification, challenges, and its future perspectives. 
The work published in Sensors, 19(18)[Brennand et al. 2019] presents the updated algorithm and methodologies used in the routing suggestion service. Among the improvements, we can mention, the implementation of the packet scheduling algorithm in the MAC layer to decrease the number of collisions and increase the delivery rate. An algorithm to reduce the number of RSUs required for full scenario coverage was developed. The article also presents the new road classification system based on the Level of Service, thus giving more realistic information about the road conditions to the route suggestion algorithm. Finally, a thorough evaluation using different urban scenarios was presented and compared with several solutions in the literature, thus showing its effectiveness. FOXS shows a reduction in stop time by up to $70 \%$ and, the planning time index by up to $49 \%$. When considering communication evaluation metrics, FOXS reaches a better result than other solutions on the packet collisions metric (up to 11.5\%) and the application delay metric (up to $30 \%$ ).

Finally, a service that uses a hybrid VANET communication and Fog computing paradigm was proposed (in the submission process). The main contribution of this work is the development of an efficient communication protocol in which it will allow even vehicles that are far from a Cloudlet to receive and send information about their routes. Results show that the service's proposed multihop communication protocol reduces network congestion by more than $90 \%$, maintaining the coverage in $97 \%$ in different scenery characteristics. Regarding traffic efficiency, traffic quality is increasing by $19 \%$, reducing carbon emissions by $10 \%$.

\section{References}

Ai, Y., Peng, M., and Zhang, K. (2018). Edge computing technologies for internet of things: a primer. Digital Communications and Networks, 4(2):77-86.

Al-Sultan, S., Al-Doori, M. M., Al-Bayatti, A. H., and Zedan, H. (2014). A comprehensive survey on vehicular ad hoc network. Journal of network and computer applications, 37:380-392.

Bonomi, F., Milito, R., Zhu, J., and Addepalli, S. (2012). Fog computing and its role in the internet of things. In Proceedings of the first edition of the MCC workshop on Mobile cloud computing, pages 13-16. ACM.

Brennand, C. A., Boukerche, A., Meneguette, R., and Villas, L. A. (2017). A novel urban traffic management mechanism based on fog. In Computers and Communications (ISCC), 2017 IEEE Symposium on, pages 377-382. IEEE.

Brennand, C. A., Cunha, F., Maia, G., Cerqueira, E., Loureiro, A. A., and Villas, L. A. (2016). Fox: A traffic management system of computer-based vehicles fog. In 2016 IEEE Symposium on Computers and Communication (ISCC), pages 1-6. IEEE.

Brennand, C. A., de Souza, A. M., Maia, G., Boukerche, A., Ramos, H., Loureiro, A. A., and Villas, L. A. (2015). An intelligent transportation system for detection and control of congested roads in urban centers. In Computers and Communication (ISCC), 20th IEEE Symposium on, pages 476-481. IEEE.

Brennand, C. A., Geraldo Filho, P. R., Maia, G., Cunha, F., Guidoni, D. L., and Villas, L. A. (2019). Towards a fog-enabled intelligent transportation system to reduce traffic jam. Sensors, 19(18):3916. 
Cookson, G. and Pishue, B. Inrix global traffic scorecard. inrix research (2019).

Cookson, G. and Pishue, B. (2017). Inrix global traffic scorecard.

da Cunha, F. D., Villas, L., Boukerche, A., Maia, G., Viana, A. C., Mini, R. A. F., and Loureiro, A. A. F. (2016). Data communication in vanets: Protocols, applications and challenges. Ad Hoc Networks, 44:90-103.

De Souza, A. M., Brennand, C. A., Yokoyama, R. S., Donato, E. A., Madeira, E. R., and Villas, L. A. (2017). Traffic management systems: A classification, review, challenges, and future perspectives. International Journal of Distributed Sensor Networks, 13(4):1550147716683612.

de Souza, A. M., Yokoyama, R. S., Maia, G., Loureiro, A., and Villas, L. (2016). Realtime path planning to prevent traffic jam through an intelligent transportation system. In Computers and Communication (ISCC), 2016 IEEE Symposium on, pages 726-731.

El-Sayed, H. and Chaqfeh, M. (2019). Exploiting mobile edge computing for enhancing vehicular applications in smart cities. Sensors, 19(5):1073.

Gomides, T. S., Lourenço, M., de Souza, P. H., and Guidoni, D. L. (2019). Sgtd: Sistema de gerenciamento de tráfego distribuído para redes veiculares. In Anais do XXXVII Simpósio Brasileiro de Redes de Computadores e Sistemas Distribuídos, pages 10421055. SBC.

Jeong, J., Jeong, H., Lee, E., Oh, T., and Du, D. H. (2016). Saint: Self-adaptive interactive navigation tool for cloud-based vehicular traffic optimization. IEEE Transactions on Vehicular Technology, 65(6):4053-4067.

Kuang, Z., Li, L., Gao, J., Zhao, L., and Liu, A. (2019). Partial offloading scheduling and power allocation for mobile edge computing systems. IEEE Internet of Things Journal.

Ling, C. W., Datta, A., and Xu, J. (2018). A case for distributed multilevel storage infrastructure for visual surveillance in intelligent transportation networks. IEEE Internet Computing, 22(1):42-51.

Papadimitratos, P., De La Fortelle, A., Evenssen, K., Brignolo, R., and Cosenza, S. (2009). Vehicular communication systems: Enabling technologies, applications, and future outlook on intelligent transportation. IEEE Communications Magazine, 47(11):84-95.

Rizzo, G., Palattella, M. R., Braun, T., and Engel, T. (2016). Content and context aware strategies for qos support in vanets. In 2016 IEEE 30th International Conference on Advanced Information Networking and Applications (AINA), pages 717-723. IEEE.

Satyanarayanan, M., Bahl, P., Caceres, R., and Davies, N. (2009). The case for vm-based cloudlets in mobile computing. IEEE pervasive Computing, 8(4).

Villas, L. A., Boukerche, A., Maia, G., Pazzi, R. W., and Loureiro, A. A. (2014). Drive: An efficient and robust data dissemination protocol for highway and urban vehicular ad hoc networks. Computer Networks, 75, Part A(0):381 - 394.

Wang, T., Zhou, J., Liu, A., Bhuiyan, M. Z. A., Wang, G., and Jia, W. (2018). Fog-based computing and storage offloading for data synchronization in iot. IEEE Internet of Things Journal. 